\title{
Soy Isoflavones Attenuate Ovariectomy-Induced Bone Loss in Stroke-Prone Spontaneously Hypertensive Rats (SHRSP)
}

\author{
Takanori Teramoto, ${ }^{1,3, *}$ Yutaka Fukui, ${ }^{1,3}$ Katsumi IKeda, ${ }^{2}$ \\ and Yukio YAMORI ${ }^{1}$ \\ ${ }^{1}$ Department of Environmental Preservation and Development, ${ }^{2}$ Otsuka Department of \\ International Preventive Nutritional Medicine, Graduate School of Human and \\ Environmental Studies, University of Kyoto, Kyoto 606-8501, Japan \\ ${ }^{3}$ Fujicco Co., Ltd., Kobe 650-8558, Japan
}

(Received December 1, 1999)

\begin{abstract}
Summary We observed the effect of two soy isoflavones, i.e., daidzin, genistin, and soy extracts on femoral density and urinary bone resorption markers in ovariectomized stroke-prone spontaneously hypertensive rats (OVXSHRSP). Experiment 1: Twenty-five SHRSP, which were fed various soy isoflavones from the age of 5 weeks, were ovariectomized at the age of 19 weeks. Then 3 weeks later, the rats were sacrificed to examine their femurs. Experiment 2: Thirty-five SHRSP received an ovariectomy at the age of 19 weeks, and then various soy isoflavones were given for 2 weeks after the surgery. Twenty-four hour urine was collected before and for 2 weeks after ovariectomy. Femoral density in genistin- or soy extract-treated OVXSHRSP was significantly higher than in untreated OVXSHRSP. Urinary bone resorption markers, pyridinoline and deoxypyridinoline in OVXSHRSP fed genistin, daidzin, or soy extracts were significantly lower than those in untreated OVXSHRSP. These results indicate that soy isoflavones attenuate bone loss in OVXSHRSP.
\end{abstract}

Key Words: SHRSP, soy isoflavone, bone loss, bone density, bone resorption marker

The continual loss of bone mass in the elderly is a natural process of aging. In women, who have a lower peak bone mass and higher incidence of osteoporotic fractures than do men at a similar age, postmenopausal estrogen deficiency appears also to be associated with increased risk of fractures [1]. Recent epidemiological studies have indicated that osteoporosis-related fractures are lower in Asia than in the Western world, possibly due to the phytoestrogen-rich soybean products consumed in large quantities by Asians [2, 3].

\footnotetext{
* To whom correspondence should be addressed.
} 
Phytoestrogens are known to be the biologically active plant compounds with both estrogenic and antiestrogenic properties [4]. Isoflavones are a group of the most important phytoestrogens, and are mostly derived from soybeans and soybean products [5].

In the present study, to examine the potential role of isoflavones in postmenopausal bone loss, we investigated the effects of two prominent soy isoflavones, daidzin and genistin, and their mixture extracted from soybeans, on bone density and urinary bone resorption markers, using ovariectomized female strokeprone spontaneously hypertensive rats (OVXSHRSP). The SHRSP has been recognized as a model for postmenopausal osteoporosis [6].

\section{MATERIALS AND METHODS}

Animals and diets. In the first study, 25 female SHRSP/Izm were supplied at the age of 4 weeks from the Disease Model Cooperative Research Association, Kyoto, Japan. On arrival, the rats were housed in an environmentally controlled laboratory room, and were acclimated to a standard laboratory diet (Powdered Funabashi SP diet, Funabashi Farm, Chiba, Japan) for 1 week. After having become accustomed to the diet, the rats were divided by initial body weight into 5 groups of 5 rats each. Rats in groups 1 and 2 were fed the standard laboratory diet and those in groups 3, 4, and 5 were fed daidzin (D), genistin (G), or soy extract (S) (Fuji-flavone P-10, Fujicco Co., Ltd., Hyogo, Japan, see Table 1), respectively, mixed with the standard laboratory diet. Each isoflavone included in these diets was restricted to a standard laboratory diet of $0.16 \mathrm{mmol} / 100 \mathrm{~g}$, so as to accomplish approximately equal intake. All rats were allowed free access to water and to experimental diets. At the age of 19 weeks, rats in group 1 (Sham) had a sham operation and those in groups $2(\mathrm{OVX}), 3(\mathrm{OVX}+\mathrm{D}), 4(\mathrm{OVX}+\mathrm{G})$, and $5(\mathrm{OVX}+\mathrm{S})$ underwent ovariectomy under pentobarbital anesthesia. Three weeks after surgery, all rats were sacrificed, and their femurs collected for further analysis.

In the second study, 35 female SHRSP/Izm were divided into 5 groups of seven rats each at the age of 18 weeks. All rats had a sham operation or underwent ovariectomy at the age of 19 weeks. Isoflavone administration was initiated following surgery. For analysis of urinary bone resorption markers, 24-h urine samples of rats were collected by using metabolic cages before and for 2 weeks after

\begin{tabular}{lc} 
Table 1. Isoflavone content of soy extracts. \\
\hline \multicolumn{1}{c}{ Isoflavone } & $\mathrm{mmol} / 100 \mathrm{~g}$ \\
\hline Daidzin & 13.76 \\
Daidzein & 0.27 \\
Genistin & 2.81 \\
Genistein & 0.11 \\
Total isoflavone & 16.95 \\
\hline
\end{tabular}


surgery. The procedures of dietary administration, group division and operation performed in this study were the same as described for the first study.

The Animal Ethics Committee of the Graduate School of Environmental Studies at the University of Kyoto approved the use of rats in these experiments.

Analytical procedures. Bone density: The right femurs removed from the rats were cleaned of soft tissue, and then their bone densities were measured by a pycnometer based on Archimedes' principle [7].

Urinary bone resorption markers: 24-h urinary bone resorption markers, pyridinoline and deoxypiridinoline, were measured by HPLC [8].

Statistical analysis. All values were expressed as the mean \pm SEM. One-way analysis of variance (ANOVA) with Fisher's post-hoc test was used for statistical comparison. $p<0.05$ was considered statistically significant.

\section{RESULTS}

\section{Food intake and body weight}

From 5 to 19 weeks of age, rats in the five groups had similar food intakes and mean body weights. Following surgery, the OVX group had a significantly higher food intake than the Sham group, and consequently the OVX group at 22 weeks of age had a significantly higher mean body weight than the Sham group (Table 2). The food intakes and the mean body weights of isoflavone-treated groups i.e., $\mathrm{OVX}+\mathrm{D}, \mathrm{OVX}+\mathrm{G}$, and $\mathrm{OVX}+\mathrm{S}$, were higher than those of the Sham group, but the differences were not significant (Table 2).

\section{Femoral density}

Figure 1 shows the femoral densities of each experimental group. Rats in the Sham, OVX $+\mathrm{G}$, or $\mathrm{OVX}+\mathrm{S}$ groups had significantly higher mean femoral densities compared with those in the OVX group $(p<0.005,0.05$, or 0.05$)$. The $\mathrm{OVX}+\mathrm{D}$ group had also a higher mean femoral density than the OVX group, but the difference was not significant $(p=0.124)$.

Table 2. Effect of ovariectomy (OVX), daidzin (D), genistin (G), and soy extract (S) on food intake and body weight in rats.

\begin{tabular}{|c|c|c|c|c|c|}
\hline Measure & Sham & OVX & $\mathrm{OVX}+\mathrm{D}$ & $\mathrm{OVX}+\mathrm{G}$ & $\mathrm{OVX}+\mathrm{S}$ \\
\hline \multicolumn{6}{|l|}{ Food intake (g/day) } \\
\hline Before surgery (5-19 weeks) & $11.7 \pm 0.4$ & $11.9 \pm 0.4$ & $11.7 \pm 0.3$ & $11.8 \pm 0.3$ & $11.6 \pm 0.3$ \\
\hline After surgery (20-22 weeks) & $11.5 \pm 0.2^{\mathrm{a}}$ & $13.4 \pm 1.1^{\mathrm{b}}$ & $12.4 \pm 1.0^{\mathrm{ab}}$ & $12.6 \pm 0.9^{a b}$ & $12.6 \pm 0.6^{\mathrm{ab}}$ \\
\hline \multicolumn{6}{|l|}{ Body weight $(\mathrm{g})$} \\
\hline Initial (at 5 weeks) & $74.0 \pm 1.4$ & $76.0 \pm 2.8$ & $80.0 \pm 1.4$ & $77.6 \pm 1.3$ & $80.4 \pm 2.9$ \\
\hline Before surgery (at 19 weeks) & $184.6 \pm 1.5$ & $185.2 \pm 3.6$ & $185.8 \pm 2.6$ & $188.4 \pm 2.7$ & $186.0 \pm 3.3$ \\
\hline Final (at 22 weeks) & $192.8 \pm 3.8^{\mathrm{a}}$ & $206.8 \pm 4.2^{\mathrm{b}}$ & $199.0 \pm 2.2^{\mathrm{ab}}$ & $199.2 \pm 2.3^{\mathrm{ab}}$ & $198.0 \pm 6.1^{\mathrm{ab}}$ \\
\hline
\end{tabular}

Vol. 28, No. 1, 2000 


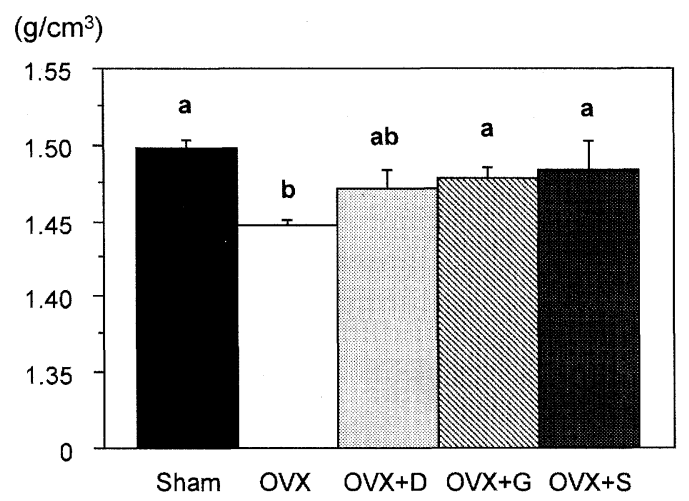

Fig. 1. Femoral densities of sham-operated (Sham), ovariectomy (OVX), OVX + daidzin $(\mathrm{OVX}+\mathrm{D}), \mathrm{OVX}+$ genistin $(\mathrm{OVX}+\mathrm{G})$, and $\mathrm{OVX}+$ soy extract $(\mathrm{OVX}+\mathrm{S})$ rats. Values are means $\pm \mathrm{SEM}, n=5$ per group. Values with different superscripts are significantly different, $p<0.05$.

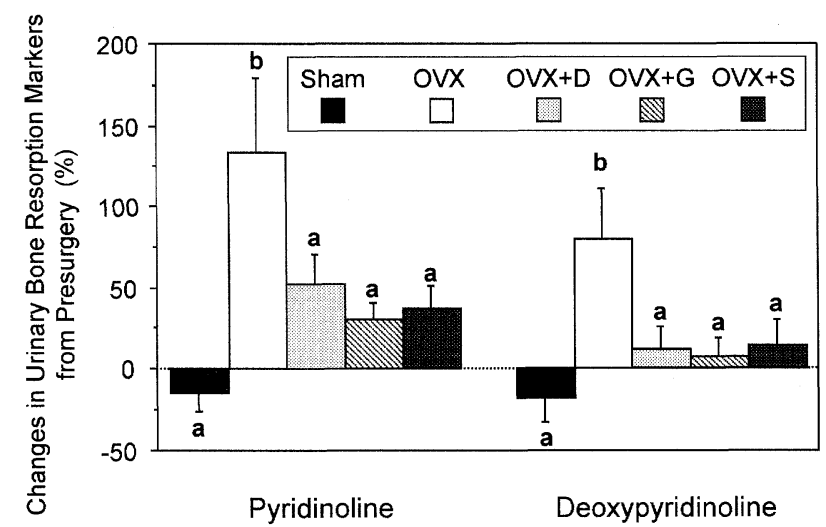

Fig. 2. Changes in urinary bone resorption markers, pyridinoline and deoxypyridinoline, of sham-operated (Sham), ovariectomy (OVX), OVX + daidzin (OVX +D), OVX+ genistin $(\mathrm{OVX}+\mathrm{G})$, and $\mathrm{OVX}+$ soy extract $(\mathrm{OVX}+\mathrm{S})$ rats. Values are given as percentage changes standardized by initial ones and are presented as means $\pm \mathrm{SEM}, n=$ 7 per group. Values with different superscripts are significantly different, $p<0.05$.

\section{Urinary bone resorption markers}

Figure 2 shows the changes in the urinary bone resorption markers, pyridinoline and deoxypyridinoline, which were standardized according to each presurgery value and presented as percentage changes. The alterations in urinary pyridinoline and deoxypyridinoline prior to and following the 2-week feeding period were significantly greater in the OVX group than in the Sham, OVX $+\mathrm{D}, \mathrm{OVX}+\mathrm{G}$, or $\mathrm{OVX}+\mathrm{S}$ groups (pyridinoline: $p<0.005,0.05,0.005$, or 0.005 , deoxypyridinoline: $p<0.005,0.05,0.05$, or 0.05 ). 


\section{DISCUSSION}

The present study demonstrates that the oral administration of soy isoflavones - daidzin, genistin, and soy extracts - was associated with the attenuation of ovariectomy-induced bone loss in SHRSP. Assuming a causal relationship, the mechanism seems to depend, at least in part, on the suppression of bone resorption. Bone resorption markers, pyridinoline and deoxypyridinoline, are nonreducible crosslinks released from bone during bone resorption [9].

Previous studies performed in rats and humans indicate that estrogen treatment inhibits the increase of urinary bone resorption marker and attenuates the reduction of bone loss [10-12]. It is also known that isoflavones show estrogenlike activity. Accordingly, the bone-protective effect of isoflavones is considered to be similar to that of estrogen.

In this study, the administration of genistin and of soy extracts containing genistin were associated with somewhat more effective reduction in bone loss than that of daidzin. It has been shown that ingested genistin and daidzin are converted to their aglycons, genistein and daidzein, in the gastrointestinal tract and that they then possess effective estrogenic activity [13]. In vitro experiments using several phytoestrogens confirmed that genistein had a slightly higher estrogenic activity than daidzein $[14,15]$. Other in vitro experiments on rat bone tissues indicated that genistein inhibited parathyroid hormone-stimulated bone resorption but increased alkaline phosphatase activity, the latter being a potential marker of bone formation [16]. Moreover, it has been reported that genistein suppresses osteoclastic activity in vitro and in vivo owing to its specific tyrosine kinase inhibitory action [17]. Further studies are needed to confirm whether or not the action of genistein in preventing bone loss is different from that of daidzein.

After ovariectomy, the food intake was slightly inhibited and the body weight gain was lower in the isoflavone-treated rats than in the non-treated OVX rats. Similar but significant inhibitions were noted with respect to estrogen treatment for ovarian hormone deficiency $[18,19]$. Current observations indicate that estrogen, via the regulation of leptin production, is related to the regulation of feeding behavior [20]. Isoflavones possess weak estrogenic activity and thus they may have a similar effect on the regulation of leptin production.

From these results we can conclude that oral soy isoflavones are associated with attenuation of bone loss induced by ovariectomy in female SHRSP, and these results suggest the possibility that soybean isoflavones may contribute to the prevention of postmenopausal osteoporosis and obesity in women.

\section{REFERENCES}

1. Albright, F., Smith, P.H., and Richardson, A.M. (1941): Postmenopausal osteoporosis: Its clinical features. J. Am. Med. Assoc., 116, 2465-2474.

Vol. 28, No. 1, 2000 
2. Kao, P.C., and P'eng, F.K. (1995): How to reduce the risk factors of osteoporosis in Asia. Chin. Med. J., 55, 209-213.

3. Ho, S.C., Bacon, W.E., Harris, T., Looker, A., and Maggi, S. (1993): Hip fracture rates in Hong Kong and the United States, 1988 through 1989. Am. J. Public Health, 83, 694-697.

4. Adlercreutz, H., and Mazur, W. (1997): Phyto-oestrogens and Western diseases. Ann. Med., 29, 95-120.

5. Coward, L., Barnes, N.C., Setchell, K.D.R., and Barnes, S. (1993): The antitumor isoflavones, genistein and daidzein, in soybean foods of American and Asian diets. J. Agric. Food Chem., 41, 1961-1967.

6. Yamori, Y., Fukuda, T., Tsuchikura, K., Ikeda, K., Nara, Y., and Horie, R. (1991): Stroke-prone SHR (SHRSP) as a model for osteoporosis. Clin. Exp. Hypertens. A, 13, 755 762 .

7. Kalu, D.N., Liu, C.C., Salerno, E., Hollis, B.W., Echon, R., and Ray, M. (1991): Skeletal response of ovariectomized rats to low and high doses of $17 \beta$-estradiol. Bone Miner., 14, $175-187$.

8. Takahashi, M., Ohishi, T., Aoshima, H., Kushida, K., Inoue, T., and Horiuchi, K. (1993): Prefractionation with cation exchanger for determination of intermolecular crosslinks, pyridinoline and pentosidine, in hydrolysate. J. Liq. Chromatogr., 16, 1355-1370.

9. Kent, G.N. (1995): Markers of bone resorption and their application in metabolic bone disease. Clin. Biochem. Rev., 16, 4-10.

10. Goulding, A., Gold, E., and Lewis, B.N.J. (1996): Effects of hysterectomy on bone in intact rats, ovariectomized rats, and ovariectomized rats treated with estrogen. J. Bone Miner. Res., 11, 977-983.

11. Prestwood, K.M., Pilbeam, C.C., Burleson, J.A., Woodiel, F.N., Delmas, P.D., Deftos, L.J., and Raisz, L.G. (1994): The short-term effects of conjugated estrogen on bone turnover in older women. J. Clin. Endocrinol. Metab., 79, 366-371.

12. Chen, J.T., Hosoda, K., Hasumi, K., Ogata, E., and Shiraki, M. (1996): Serum N-terminal osteocalcin is a good indicator for estimating responders to hormone replacement therapy in postmenopausal women. J. Bone Miner. Res., 11, 1784-1792.

13. Murkies, A. (1998): Phytoestrogens-What is the current knowledge? Aust. Fam. Physician, 27, S47-S51.

14. Markiewicz, L., Garey, J., Adlercreutz, H., and Gurpide, E. (1993): In vitro bioassays of non-steroidal phytoestrogens. J. Steroid Biochem. Mol. Biol., 45, 399-405.

15. Miksikek, R.J. (1994): Interaction of naturally occurring nonsteroidal estrogens with expressed recombinant human estrogen receptor. J. Steroid Biochem. Mol. Biol., 49, 153-160.

16. Yamaguchi, M., and Gao, Y.H. (1998): Inhibitory effect of genistein on bone resorption in tissue culture. Biochem. Pharmacol., 55, 71-76.

17. Blair, H.C., Jordan, S.E., Peterson, T.G., and Barnes, S. (1996): Variable effects of tyrosine kinase inhibitors on avian osteoclastic activity and reduction of bone loss in ovariectomized rats. J. Cell. Biochem., 61, 629-637.

18. Bahram, H.A., Dilshad, A.K., Shanil, S.J., and Alvar, S. (1997): The ovarian hormone deficiency-induced hypercholesterolemia is reversed by soy protein and the synthetic isoflavone, ipriflavone. Nutr. Res., 17, 885-894.

19. Roudebush, R.E., Magee, D.E., Benslay, D.N., Bendele, A.M., and Bryan, H.U. (1993): Effect of weight manipulation on bone loss due to ovariectomy and the protective effects of estrogen in the rats. Calcif. Tissue Int., 53, 61-64.

20. Shimizu, H., Shimomura, Y., Nakanishi, Y., Futawatari, T., Ohtani, K., Sato, N., and Mori, M. (1997): Estrogen increases in vivo leptin production in rats and human subjects. $J$. Endocrinol., 154, 285-292. 